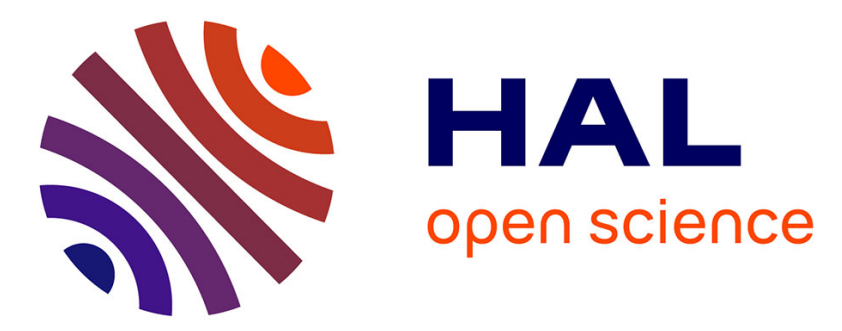

\title{
L'abeille dans le géosystème: essai de cartographie des ressources mellifères
}

\author{
Gérard Briane, Jean-Luc Cabrol
}

\section{To cite this version:}

Gérard Briane, Jean-Luc Cabrol. L'abeille dans le géosystème: essai de cartographie des ressources mellifères. Revue Géographique des Pyrénées et du Sud-Ouest, 1986, L'élément et le système, 57 (3), pp.363-373. 10.3406/rgpso.1986.4954 . hal-02624092

\section{HAL Id: hal-02624092 \\ https://hal-univ-tlse2.archives-ouvertes.fr/hal-02624092}

Submitted on 26 May 2020

HAL is a multi-disciplinary open access archive for the deposit and dissemination of scientific research documents, whether they are published or not. The documents may come from teaching and research institutions in France or abroad, or from public or private research centers.
L'archive ouverte pluridisciplinaire HAL, est destinée au dépôt et à la diffusion de documents scientifiques de niveau recherche, publiés ou non, émanant des établissements d'enseignement et de recherche français ou étrangers, des laboratoires publics ou privés. 


\section{L'abeille dans le géosystème : essai de cartographie des} ressources mellifères

Gérard Briane, Jean-Luc Cabrol

\section{Citer ce document / Cite this document :}

Briane Gérard, Cabrol Jean-Luc. L'abeille dans le géosystème : essai de cartographie des ressources mellifères. In: Revue géographique des Pyrénées et du Sud-Ouest, tome 57, fascicule 3, 1986. L'élément et le système. pp. 363-373;

doi : https://doi.org/10.3406/rgpso.1986.4954

https://www.persee.fr/doc/rgpso_0035-3221_1986_num_57_3_4954

Fichier pdf généré le 06/04/2018 


\title{
Résumé
}

Un inventaire des plantes apicoles a été réalisé en corrélation avec l'étude des formations végétales dans un rayon de $3 \mathrm{~km}$ autour d'un rucher de la moyenne montagne pyrénéenne. Cela a contribué à mettre en évidence la distribution spatio-temporelle des ressources mellifères et d'associer celle-ci au comportement des abeilles sur une année. Ces résultats couplés aux analyses mellissopalynologiques pratiquées à différentes époques prouvent que les abeilles adoptent une stratégie variable face à leur environnement végétal, dans l'espace et dans le temps. Cette étude localisée montre la possibilité d'une cartographie de la phénologie des potentialités mellifères, à partir de l'analyse géosystémique, permettant de valoriser les secteurs apicoles et leur dynamique dans un milieu végétal souvent délaissé par l'agriculture.

\section{Resumen}

La abeja dentro del geosistema. Ensayo de cartografía de los recursos melíferos. En un radio de tres kilómetros alrededor de un colmenar situado en la media montaña del Pirineo de Haute-Garonne, se ha hecho el inventario de las plantas apícolas en estrecha relación con el estudio de las formaciones vegetales. Se ha podido diseñar la distribución espacio-temporal de estos recursos, y, relacionar con ella el comportamiento de las abejas a lo largo del año. Estos resultados, junto con el análisis de mieles y pólenes en diversas épocas del año, temuestran que las abejas adoptan, frente al entorno vegetal, une estrategia variable en el espacio y en el tiempo. Este estudio local muestra que es posible la realización de una cartografía de la fenología de las potencialidades melífera partiendo del análisis geosistémico con lo que se podría valorizar los sectores apícolas y su dinámica en medios vegetales generalmente abandonados por la agricultura.

\begin{abstract}
Bees and the geosystem : mapping melliferous resources. In a $3 \mathrm{~km}$ zone around a hive (middle pyrenean mountain, Haute Garonne) a record was created of ararian plants together with a study of the flora. This helped the illustration of the time-space distribution of melliferous resources, linked to the behaviour of bees during a year. The results compared to several mellino-palynologie analyses show that bees adopt differing strategies to their floral environment in space and time. Such a study shows the possibility of the creation of a map of the phenology of the melliferous resource, starting from a geosystematic analysis, which allows a valuation of aparian sectors and their dynamic in a vegetation often ignored by agriculture.
\end{abstract}


REVUE GÉOGRAPHIQUE DES PYRIENIEIES FT DU SUD-OUIST TOME 57, FASC. 3, pp. 363-373, Toulouse, 1986.

\title{
L'abeille dans le géosystème : essai de cartographie des ressources mellifères
}

\author{
par Gérard Briane * et Jean-Luc Cabrol *
}

Les recherches apicoles sont le plus souvent fondées sur l'étude de la biologie de l'abeille (1) ou sur son action pollinisatrice des productions végétales (2). Peu de travaux sont consacrés aux rapports entre les abeilles et leur environnement, dans un espace donné et en fonction de la phénologie des espèces végétales. La répartition et l'évolution dans le temps et dans l'espace n'a jamais été, à notre connaissance, synthétisée cartographiquement. Un tel travail devrait pourtant permettre aux apiculteurs une meilleure exploitation du milicu en favorisant les transhumances de ruchers ou en définissant plus sûrement des « crus " de micl. L'ćtude de la répartition spatio-temporellc des ressources mellifères restait donc à entreprendre (3). L'objectif premier résidait dans l'analyse détaillée de l'aire de butinage d'un rucher (15 ruches) installé au lieu-dit "Peyrille», commune de Fougaron. Haute-Garonne (4).

\section{Des géosystèmes de moyenne montagne humide en voie d'enfrichement}

Le secteur d'étude correspond au rayon d'action d'une abeille butineuse, qui varie de 1,5 à $3 \mathrm{~km}$ autour d'une ruche, en fonction du

(*) Etudiants de troisième cycle, Université de Toulouse-Le Mirail, (CIMA-UA 366, CNRS), 31058 Toulouse Cedex.

(1) Cf. Chauvin, 1968, Frich, 1969.

(2) Cf. Louveaux, 1968, Louveaux et Pesson, 1984.

(3) En fait, cette étude a été lancée il y a quelques années par un apiculteur ariégeois, M. Philippe Cresto, aujourd'hui décédé, qui souhaitait réaliser une thèse sur ce thème en animant tout un réseau d'apiculteurs contrôlant des ruchers témoins.

(4) Cf. Briane et Cabrol, 1985. 
relief local; c'est un petit bassin versant du Massif de l'Arbas relativement fermé, entre $400 \mathrm{~m}$ et $1400 \mathrm{~m}$ d'altitude, encadré par des massifs montagneux (Mt Aragnoué au nord, Crête de l'Homme Mort à l'est, le Tuc de Piscatelle au sud). C'est un milieu humide (1 $053 \mathrm{~mm}$ de précipitations moyennes annuelles) et assez nébuleux de la façade nord-pyrénéenne. D'après la carte de végétation de la France au $1 / 200000^{\circ}$ (Foix), on note, dans les différents étagements, trois séries de végétation: deux de chênes (pubescent et pédonculé, où il $\mathbf{y}$ a un faciès à châtaignier) et une série du hêtre.

Dans ce secteur autrefois entièrement cultivé, sauf sur l'ombrée restée forestière, l'occupation du sol peut aujourd'hui se résumer ainsi depuis l'abandon progressif des cultures: large prédominance des forêts (moins de $60 \%$ dont $30 \%$ de hêtraie sur la surface étudiée), progression constante des friches $(20 \%)$, prairies de fauche et pâturées $(19 \%)$, cultures et jardins $(0,5 \%)$, et des éléments linéaires dont on verra l'importance sur le plan mellifère.

Envisager de la sorte les formations végétales autorise une analyse plus détaillée à partir de la reconnaissance et de la cartographie des géosystèmes et des géofaciès. Elle pourrait s'opérer à partir de la distinction de l'espace forestier, de l'espace pastoral, de l'espace cultivé, de l'espace linéaire pour mettre en évidence des groupements ou faciès mellifères caractéristiques (5).

\section{De l'aire de butinage à l'évaluation des ressources mellifères}

La cartographie au $1 / 10000^{*}$ des phénomènes apicoles nécessiterait, en premier lieu, la distinction des principales formations végétales. Cela présente l'avantage de mieux saisir la dynamique des milieux et de permettre d'envisager des "types de formations végétales apicoles" sur l'aire déterminée. Par ailleurs, cette pratique autorise une généralisation ultérieure de ce type d'étude à des échelles différentes.

La connaissance des ressources mellifères passe par un inventaire des espèces apicoles (6) sur l'aire déterminée et sur une année. Dans un fichier ont été regroupés l'époque et la durée de floraison des différentes espèces, leur localisation au sein des formations végétales, leur abondance, leur attractivité et leur intérêt apicole. Plus de 170 espèces ont été recensées, dont l'intérêt de chacune peut varier pour les abeilles comme pour les apiculteurs. Certaines espèces sont butinées pour la constitution du miel; ce sont les plantes mellifères au sens strict, qui fournissent nectar et miellat, ou les deux. Le nectar est sécrété par les nectaires de la plante elle-même alors que le miellat résulte généralement de sucres rejetés par les pucerons parasites de la plante (cas de l'érable, Acer campestre). Dautres espèces végéta-

(5) Cf. Bertrand, 1978.

(6) Cf. Alphandéry, 1935, Bonnier, Costes, 1937, Rabiet, 1981. 
les ne servent qu'à la nourriture des abeilles et des larves et n'entrent pas dans l'élaboration du miel: ce sont des plantes pollinifères, procurant uniquement du pollen aux abeilles (cas du noisetier, Corylus avellana). Enfin, d'autres végétaux sont utilisés par la colonie pour l'amélioration des structures de la ruche ou comme élément bactéricide: dans ces cas là, ces espèces fournissent de la propolis, à partir de la gomme des bourgeons, de la résine, de la séve d'écorce (cas du tremble, Populus tremula). En outre, certaines plantes apportent plusieurs de ces éléments à la fois : les tilleul:; (Tilia sp.) : nectar et miellat, les saules (Salix sp.) : nectar et pollen, le châtaignier (Castanea sativa): nectar, pollen et miellat, etc.

A ces facteurs déjà complexes, il conviendrait d'en ajouter bien d'autres pour une étude plus complète, à savoir : la densité des plantes, leur attractivité spécifique pour les abeilles, la quantité et la qualité de nectar sécrété par la plante, l'accessibilité des nectaires, les fluctuations journalières de la sécrétion nectarifère, les quantités de pollens et de miellat disponib'es, etc., la variabilité régionale de chaque espèce (beaucoup d'espèces nectarifères en montagne ne le sont pas en plaine), la diversité spécifique variable au cours des saisons, le rôle des races d'abeilles (variation de la longueur de leur langue atteignant plus ou moins facilement les nectaires), l'influence du milieu (climat, altitude, exposition, sol, etc.).

Cette diversité d'éléments entrant en jeu rend bien compte de la difficulté d'appréhender globalement ces phénomènes.

\section{Esquisse d'une cartographie phénologique des ressources mellifères}

Le cadre de cette étude ne permettait pas de prendre en compte la totalité de ces facteurs dans une carte. Le choix s'est donc porté sur les espèces apicoles les plus abondantes et les plus attractives ou les deux. Cette sélection opérée, il a fallu ébaucher des cartes de potentialités mellifères traduisant bien la phénologie des espèces. Mais ces cartes ne pouvaient correspondre aux simples saisons : il a été nécessaire de déterminer des "saisons mcllifères" où la biologie et le comportement des abeilles coïncident globalement avec la phénologie de ces plantes. Ainsi quatre cartes représentent-elles les quatre saisons mellifères.

\section{De Février à Mars : le démarrage du couvain.}

Dès les premiers beaux jours, la reine commence à pondre; les ouvrières reconstituent les réserves de pollen (très périssables) en exploitant les moindres ressources alors disponibles: elles butinent des espèces à la fois nectarifères et pollinifères (saules) ou très souvent, des plantes exclusivement pollinifères (noisetier; buis: Buxus 
sempervirens, aulne : Alnus glutinosa); les abeilles se procurent enfin de la propolis sur les trembles (carte A).

La carte révèle que le milieu végétal n'est pas exploitable sur toute sa surface par les abeilles et que seuls des secteurs précis recèlent des potentialités mellifères: des secteurs linéaires (bords de routes, lisières de friches hautes, bords des eaux, haies, etc., comprenant principalement différentes espèces de saules et le noisetier), des masses homogènes (friches, taillis) qui accueillent abondamment le buis sur substratum calcaire, des secteurs ponctuels (bords de chemins, jardins, friches) où se retrouvent des espèces apicoles peu abondantes: prunelier (Prunus spinosa) et tremble.

\section{D'Avril à Mai : la constitution de la ruche.}

Les jeunes abeilles butineuses sortent en grand nombre pour faire face aux nombreux besoins de la colonie. les larves consomment du pollen et les abeilles du nectar pour assurer leur nourriture. La diversité des espèces végétales apicoles en voie de floraison augmente et leur distribution s'étend dans le milieu végétal (carte $B$ ).

En forêt, deux espèces arborées sont disséminées sur de vastes étendues: le merisier (Cerasus avium), qui fournit nectar et pollen, et l'érable champêtre, qui n'apporte que du nectar. Les friches hautes recèlent de nombreuses espèces mellifères: différentes aubépines (Crataegus sp.), la bourdaine (Rhamnus frangula), le cornouiller sanguin (Cornus sanguinea, etc.). Dans les prairies, les butineuses peuvent rencontrer le pissenlit (Taraxacum dens-leonis), le lotier (Lotus corniculatus), la cardamine des près (Cardamine pratensis), etc.; ce milieu semble toutefois moins fréquenté à cette période que les pré. cédents. Enfin quelques ressources ponctuelles, surtout constituées par des arbres fruitiers sont disséminées dans les prairies, vergers et jardins.

\section{De Juin à Juillet : la miellée principale.}

Durant cette période du début d'été, les ressources mellifères sont considérables. C'est à ce moment que l'apiculteur fera la récolte de miel la plus abondante de l'année. La carte traduit bien, par la variété et la densité des phénomènes représentés, l'importance de ces potentialités (carte $C$ ).

Une masse homogène se dégage en premier lieu, constituée par le châtaignier, qui est l'essence mellifère primordiale à cette époque; de plus, des individus dont l'exposition diffère ne fleurissent pas pendant la même période : ce décalage de floraison laisse le loisir aux abeilles d'exploiter cette réserve plus longtemps; on note également la présence du miellat sur les feuilles du châtaignicr. Mais l'avenir de cette châtaigneraie semble menacé par le chancre de l'écorce (Endothia parasitica). 
Les masses de contenu hétérogène ne sont pas négligeables : les friches basses regorgent de ronces (Rubus sp.) très visitées par les abeilles; les prairies, malgré leur richesse floristique, ne sont que très peu exploitées en cette période.

Des espèces telles que tilleuls (Tilia sp.) et robinier (Robinia pseudoacacia) sont disposćes de façon ponctuelle dans les prairies, bords de routes ou bois, mais assurent cependant un rôle important de fournisseur de nectar.

Les abeilles se consacrent donc principalement à quelques espèces et négligent, voire ignorent, de multiples espèces fleuries, qui seront toutefois visitées ultérieurement.

\section{D'Août à Octobre : le pré-hivernage.}

Cette dernière période contraste avec la précédente mais rappelle Février-Mars. En effet, les ressources mellifères sont alors plus limitées et plus localisées; toutefois il existe une importante diversité d'espèces apicoles que l'on retrouve généralement distribuées de façon linéaire ou ponctuelle. Ce sont le bord des routes, le long de cours d'eau, les friches basses qui accueillent différents cirses (Cirsium sp.), l'angélique (Angelica sylvestris), l'eupatoire (Eupatorium cannabinum), la reine des prés (Spiræa ulmaria), la balsamine d'Inde (Impatiens glandulifera), etc. D'autre part, les prairies de fauche recìlent, à cette époque et de façon relativement homogène, toutes sortes de menthes (Mentha sp), de scabieuses (Scabiosa sp.), de rentaurées (Centaurea sp.), etc. Quant aux rares cultures présentes dans la vallée étudiée, seul le mais (Zea mays) fournit une source de pollen abondante et recherchée. Enfin, certains milieux ponctuels ne sont pas à négliger : c'est le cas de rares landes à bruvères (Erica vagans et Calluna vulgaris), celui du lierre (Hedera helix), dernière espèce à fleurir et qui a, ici, la particularité d'être abondant sur tous les bâtiments plus ou moins abandonnés (carte $\mathrm{D}$ ).

La région étudiée semble propice à la production de crus de miel de châtaignier et, secondairement, de ronce et de tilleul. Ce dernier, qui a un plus grand intérêt commercial, doit faire l'objet d'une analvse plus détaillée dans le temps et dans l'espace. Dans ce secteur d'étude, les abeilles visitent plus de 170 espèces de plantes dont un certain nombre est délaissé en plaine : euphorbe (Eunhorbia amygdaloides) hellébore (Helleborus foetidus). maïs, gui (Viscum album), etc. La diversité taxinomique des plantes apicoles augmente du printemps à l'été mais. parallèlement. l'attractivité spécifique tend à décroîtrc globalement durant la même période : une espèce donnée est généra. lement plus attractive au printemps que durant l'été où alle est concurrencée par de nombreuses autres espèces mellifères.

L'exploitation du milieu végétal par les abeilles est variable tout au long d'une année. Les friches disposent de fortes potentialités apicoles durant la majeure partie de l'année, hors l'hiver. Les prairies sont 

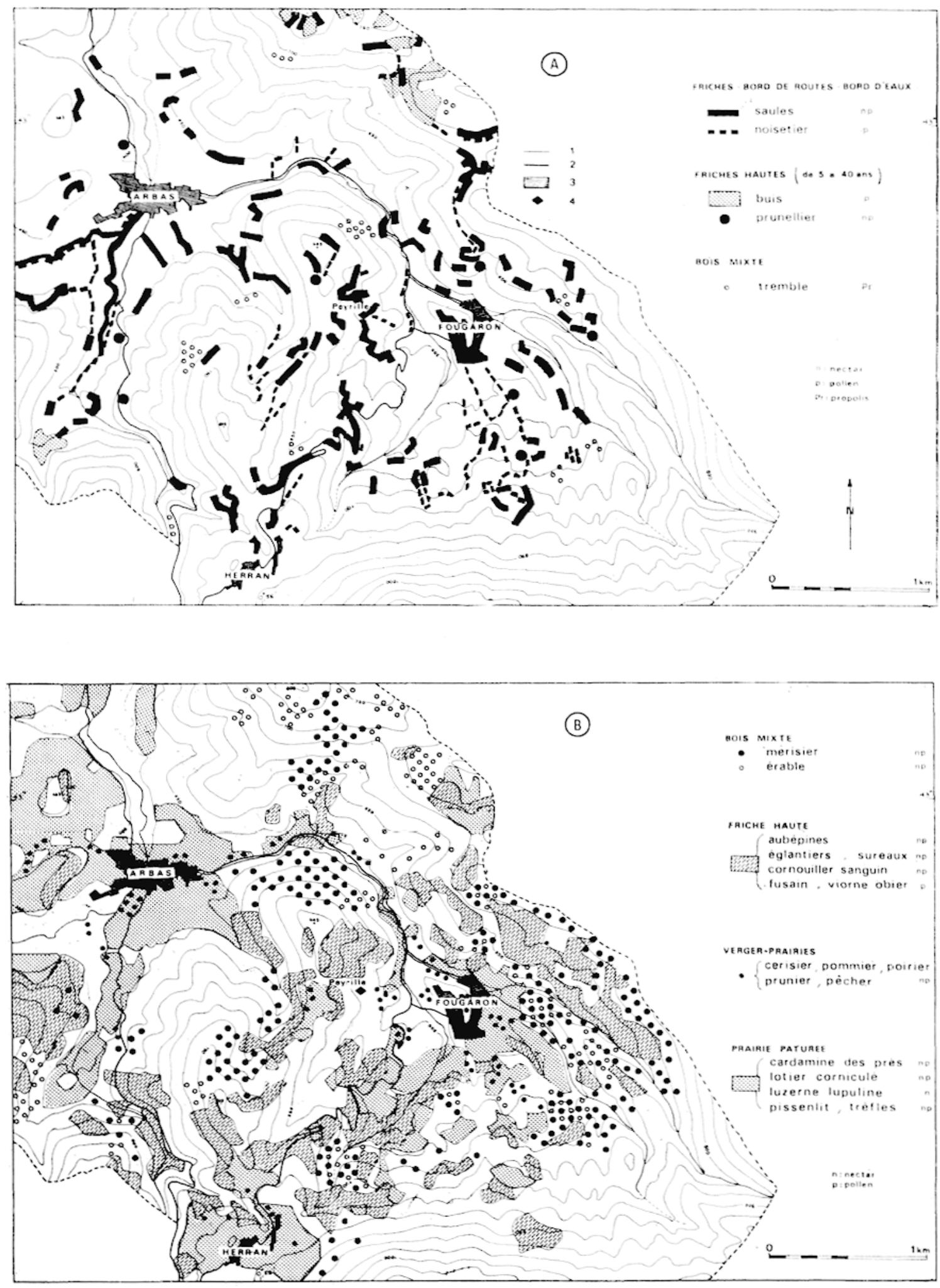

Fig. 1

Ressources mellifères du rucher de Peyrille.

A. Démarrage du couvain. - B. Constitution de la ruche. 

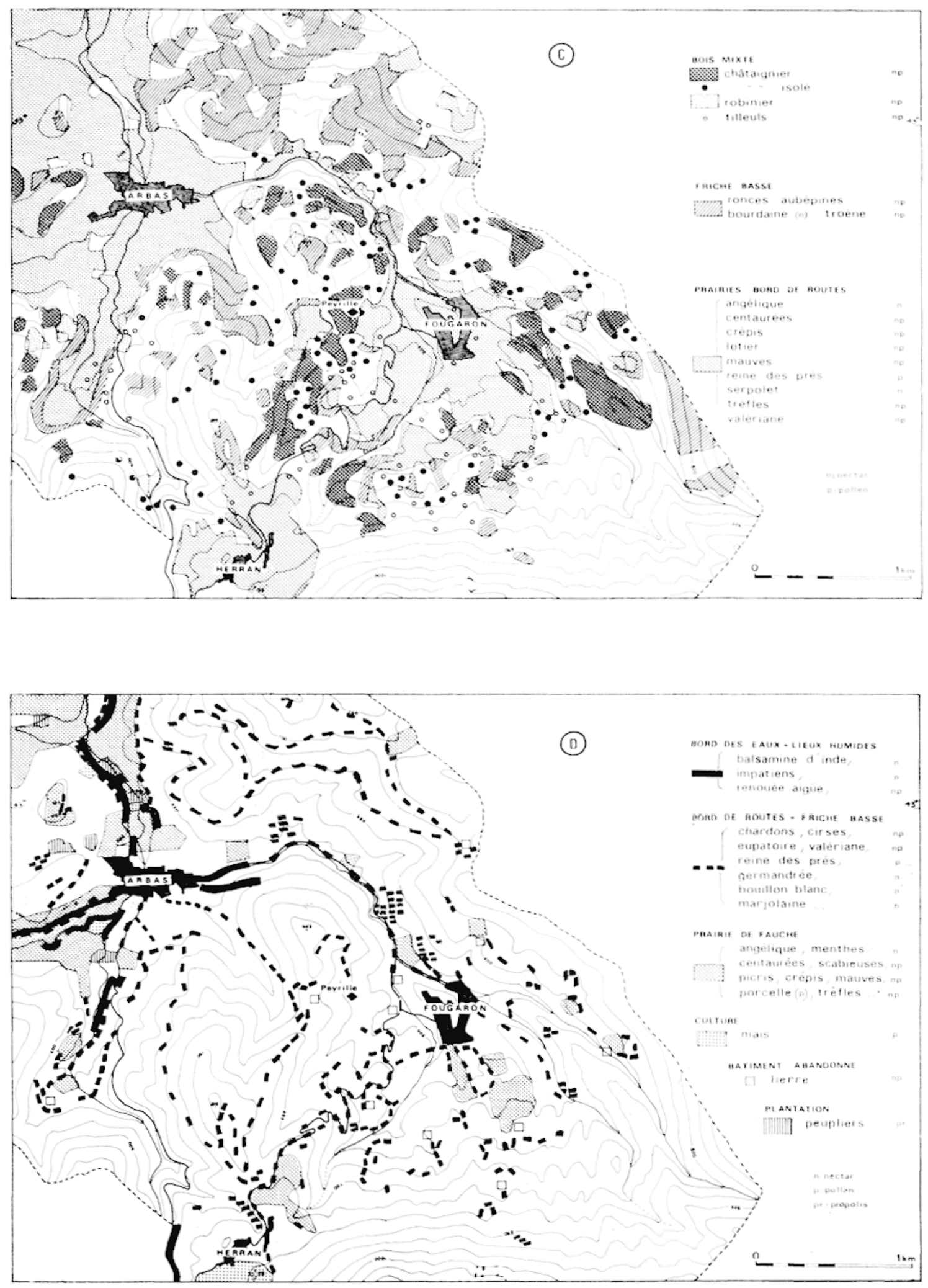

C. Miellée principale. - D. Pré-hivernage. -

Légende commune, voir carte A : 1. Ruisseau. - 2. Route. - 3. Village. 4. Rucher. 
plus ou moins attractives pendant les saisons printanière, estivale et automnale. La forêt présente un intérêt mellifère réduit à quelques espèces arborescentes au printemps et en été (sauf la hêtraie qui ne révèle, ici, presque aucune ressource apicole) (fig. 2).

Hedera helix (L)

Calluna vulgar is ( $S$ )

Teucrium scorodonia (L)

Valeriana obficinalis (L)

Origanum vulgare (L)

Polygonum cuspidatum (L)

Impatiens sp. (L)

Spiraea ulmaria (4)

Malva sp. (L)

Mentha sp. (L)

Eupatorium cannabinum (L)

Erica vagans (L)

Centaurea sp. (L)

Rubus fruticosus * (L)

Picris sp. ( $\mathrm{J}$ )

Tilia sp.* (L)

Thymus serpillum (L)

Castanea sativa * (S)

Scabiosa sp. (L)

Crepis sp. (L)

Ligustrum vulgare (L)

Cardues sp. (L)

Sambucus nigra (L)

Robinia pseudoacacia * (L)

Angelica silvestris (L)

Trifolium sp. (L)

Lamium sp. (L)

Cirsium sp. ( $T$ )

Rhamnus frangula (L)

Myosotis sp. (L)

Aquilegia vulgaris (L)

Lotus corniculatus (L)

Acer campestre * (L)

Crataegus sp. (L)

Malus communis ( $P$ )

Cerasus avium * (M)

Taraxacum dens leonis "(D)

Cardamine pratensis (L)

Buxus sempervirens (L)

Salix sp. * (L)

Conylus avellana (L)
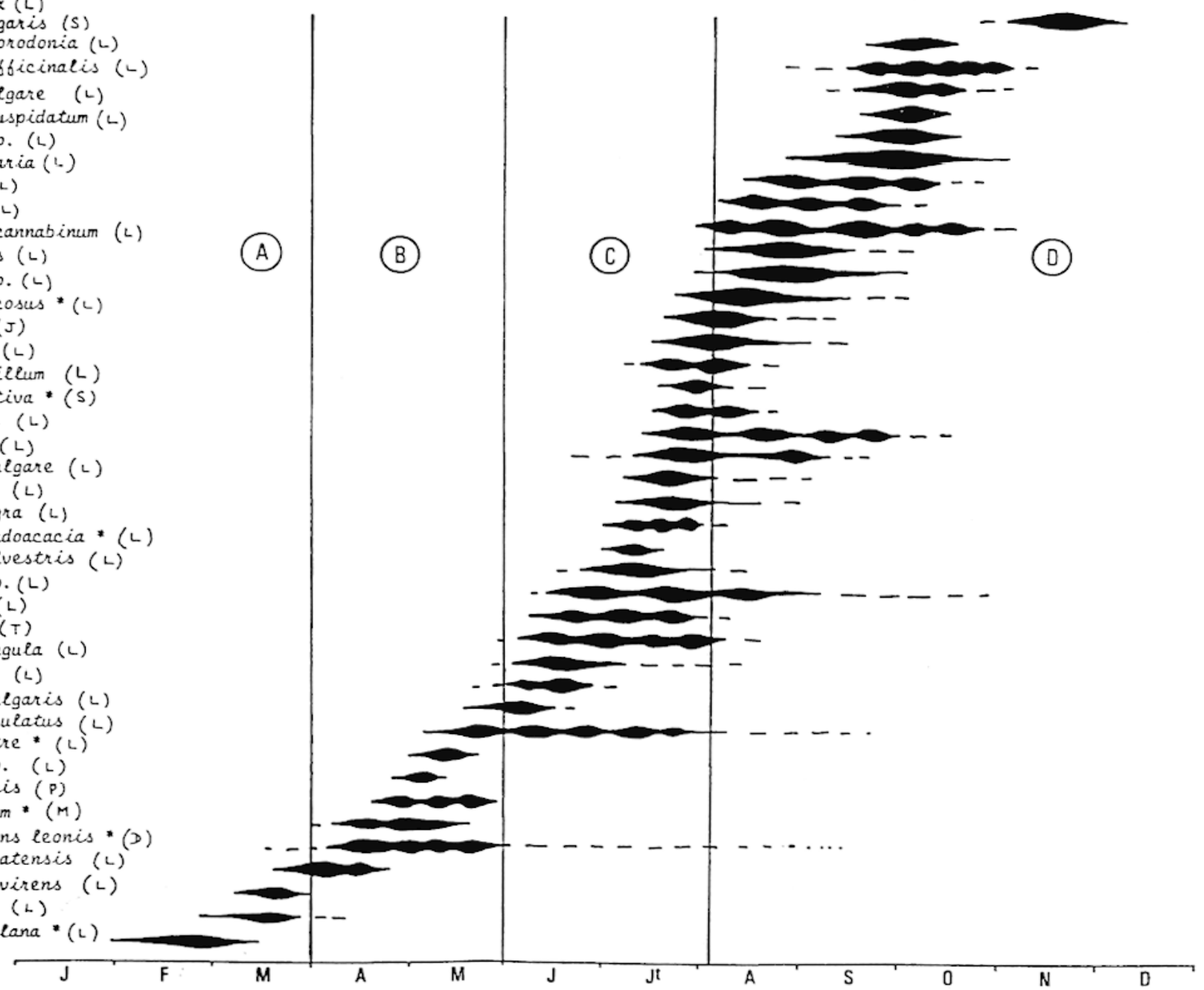

FIG. 2

La phénologie des principales plantes apicoles autour du rucher de Peyrille (1985).

\section{Apport de l'analyse mélisso-palynologique}

L'étude concomitante des pollens contenus dans le miel et des relevés de terrain est indispensable pour cléterminer la stratégie des abeilles face aux diverses potentialités mellifères offertes par l'environnement végétal. Le but de ces analyses mélisso-palynologiques (7),

(7) Analyses par Isabelle Meiffren, Labo. Botanique et Biogéo., Université Paul-Sabatier, Toulouse. 
à partir de la détermination et du calcul du pourcentage de grains de pollen issus de miels récoltés à différentes époques de l'année, est de mettre en évidence des espèces butinées (8). Elles peuvent aider à la définition de crus de miels. Par exemple, une analyse de miel prélevé dans les rayons d'une ruche de Peyrille à la fin du mois de juillet montre une écrasante prédominance de grains de pollens de châtaignier $(95,12 \%)$ alors que les rosacées (ronces surtout) n'arrivent en seconde position qu'avec $4,37 \%$ de grains de pollen et les tilleuls en troisième avec un taux pollinique de $0,02 \%$. Cependant que fin juin ils représentent $7 \%$ de l'analyse effectuée, ce qui est relativement important vue la pauvreté pollinique des miels purs de tilleul.

\section{Une recherche fondamentale et appliquée}

Cette étude, qui en est à ses débuts, doit maintenant s'affirmer aussi bien sur le plan fondamental que sur le plan de ses applications. Conçue au départ comme interdisciplinaire, et interprofessionnelle, elle a réuni des géographes, des botanistes, des palynologues, des entomologistes, des généticiens... et des apiculteurs, sans lesquels ce programme serait irréalisable, car il faut mettre en place un réseau de ruchers-témoins, au moins dans la partie centre orientale des Pyrénées.

En l'état, cette première tentative de cartographie des ressources mellifères pose plusieurs questions d'ordre méthodologique.

a. Les modèles chorologiques classiques, formations végétales ou groupements phytosociologiques, ne sont que des points de départs, indispensables mais insuffisants. Il est nécessaire de découper dans l'espace végétal des " unités »floristiques pertinentes du point de vue de la production mellifère au sens large. Ces "unités mellifères" changeant suivant les saisons et l'analyse phénologique, un peu trop souvent négligée par les différentes disciplines, deviennent ici la pierre angulaire du système apicole et de sa cartographie. De plus, dans beaucoup de cas observés, la notion même d'unité mellifère se limite à une seule espèce (ronce, robinier) qui est souvent réduite à un seul individu isolé, comme par exemple un tilleul dans un jardin, mais susceptible de fournir l'équivalent, en quantité, d'une lande de plusieurs hectares et d'accaparer tout un stade de butinage.

b. La représentation cartographique s'en trouve modifiée. En dehors de la question scalaire $\left(1 / 5000^{\circ}, 1 / 10000^{\circ}\right)$ et des problèmes de généralisation cartographique, le suivi phénologique imnose une saisie et un traitement des données qui relèvent de la seule cartographie automatique (9).

(8) Cf. Lieux, 1980, et Louveaux, 1968.

(9) Test en cours, en collaboration avec Françoise Desbordes (GIP-Reclus GS Isard). 
Dans une zone de montagne aussi peu favorisée que les Pyrénées ariégeoises, l'apiculture ne peut plus être négligée. A còté d'apiculteurs amateurs possédant plusieurs dizaines de ruches, il existe des apiculteurs professionnels pratiquant la transhumance de plusieurs centaines, voire de un à trois milliers de ruches. L'inventaire des plantes et des unités végétales mellifères susceptibles de fournir des crus de miel définis revêt un intérêt socio-économique qui n'échappe pas aux responsables de l'aménagement. De ce point de vue, l'étude attire l'attention sur certaines espèces végétales peu connues ou mal localisées (robinier), mais surtout elle pose sous un jour nouveau le problème de la valorisation des friches, ou du moins de certains de leurs faciès qui ne sont pas des espaces aussi inutiles ou inutilisables qu'on le prétend.

Dans ce cas, comme dans d'autres, l'étude et la cartographie préalables des géosystèmes ont déclenché, puis accompagné et nourri des recherches finalisées sur tel élément, - unité végétale mellifère, espèce végétale mellifère, voire individu mellifère - - qui pour êtrè situé dans l'espace et la phénologie apicoles et donner lieu à unc interprétation d'intérêt général, doit être replacé à son tour dans la totalité du géosystème et de son fonctionnement au rythme de la vie des abeilles.

\section{Bibliographie}

Alet B., 1984, L'Avifaune dans les géosystèmes de Grésigne, Université de Toulouse-Le Mirail, (thèse de $3^{\circ}$ cycle, géographie).

ALPHANdèry E., 1935, Flore mellifère de la France, de la Suisse et de la Belgique, Paris, Baillières.

BERTRAND G., 1978, Le géosystème ou système territorial naturel, RGPSO, 43, 2.

BoNNIER G., 1985, Plantes médicinales, plantes mellifères, plantes utiles et nutisibles.

BRIANE G. et CABROL J.-L., 1985, Essai de cartographie des ressources mellifères du rucher de Peyrille (Fougaron, Haute-Garonne, Université de ToulouseLe Mirail), 146 pages plus 5 cartes couleurs h.t. (mémoire de maîtrise, géographie).

Chauvin R., 1968, Traité de biologie de l'abeille, Paris, Masson.

Costes A., 1937, Flore de la France, de la Corse et des contrées limitrophes, Paris, Librairie A. Blanchard.

FriciI K.V., 1969, Vie et mours des abeilles, Paris, Albin Michel.

Gaussex H., 1964, Carte de la végétation de la France au 1/200000', Feuille de Foix. Service national de la carte de la végétation.

Lifux M.H., 1980, Acetolysis applied to microscopal honey analysis, Grana, $19,67,61$, Stockholm.

Louveaux J., 1968, L'analyse pollinique des miels in Traité de Biologie de l'abeille, t. III., p. 325-262. Paris, Masson.

Louveaux J. et Pesson P., 1984, Pollinisation et productions végétales, Paris INRA. Rabiet E., 1981, Plantes mellifères, plantes apicoles. Chez l'auteur, 196 pages. 
RÉstuÉ. - Un inventaire des plantes apicoles a été réalisé en corrélation avec l'étude des formations végétales dans un rayon de $3 \mathrm{~km}$ autour d'un rucher de la moyenne montagne pyrénéenne. Cela a contribué à mettre en évidence la distribution spatio-temporelle des ressources mellifères et d'associer celle-ci au comportement des abcilles sur une annéc. Ces résultats couplés aux analyses mellisso-palynologiques pratiquées à différentes époques prourent que les abcilles adoptent une stratégie variable face à leur environnement végétal, dans l'espace et dans le temps. Cette étude localiséc montre la possibilité d'uné cartographie de la phénologie des potentialités mellifères, à partir de l'analyse géosystémique, permettant de valoriser les secteurs apicoles et leur dynamique dans un milieu végétal souvent délaissé par l'agriculture.

SIMMARY. - BEES AND THE GEOSYSTEM : MAPPING MELlifiROLS RESOLRCES. In a $3 \mathrm{~km}$ zone around a hive (middle pyrenean mountain, Haute Garonne) a record was created of ararian plants together with a study of the flora. This helped the illustration of the time-space distribution of melliferous resources, linked to the behaviour of bees during a year. The results compared to several mellinopalynologic analyses show that bees adopt differing strategies to their iloral environment in space and time. Such a study shows the possibility of the creation of a map of the phenology of the melliferous resource, starting from a geosystematic analysis, which allows a valuation of aparian sectors and their dynamic in a vegetation often ignored by agriculture.

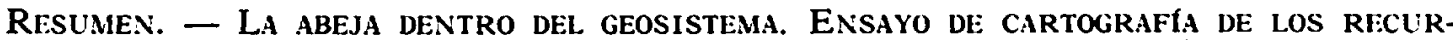
SOS MFíf́feros. En un radio de tres kilómetros alrededor de un colmenar situado en la media montaña del Pirineo de Haute-Garonne, se ha hecho el inventario de las plantas apícolas en estrecha relación con el estudio de las formaciones vegetales. Se ha podido diseñar la distribución espacio-temporal de estos recursos, y, relacionar con ella el comportamiento de las abejas a lo largo del año. Estos resultados, junto con el análisis de mieles y pólenes en diversas épocas del año, temuestran que las abejas adoptan, frente al entorno vegetal, une estrategia variable en el espacio y en el tiempo. Este estudio local muestra que es posible la realización de una cartografía de la fenología de las potencialidades melífera partiendo del análisis geosistémico con lo que se podría valorizar los sectores apícolas y su dinámica en medios vegetales generalmente abandonados por la agricultura.

Mots-Ci.́s. - Pyrénés, Pyrénées ariégeoises, massif de l'Arbas, apiculture, agriculture de montagne, zoogéographie, abeille, biogéographie, phytogéographie, plantes mellifères, mélisso-palynologie, phénologie, géosystème, cartographie, cartographie botanique, actuel. 